\title{
Complete Cells and a Complete Scientist: A Tribute to Dr. Gina Sosinsky
}

\author{
Maryann E. Martone $e^{1,2}$ \\ 1. Professor Emeritus, Department of Neurosciences and Center for Research in Biological Systems, \\ University of California San Diego, San Diego CA USA \\ 2. Director of Biosciences, hypothes.is, San Francisco, CA USA
}

It was my privilege to work for almost 20 years with Dr. Gina Sosinsky, former Council member for MSA, at UCSD at the National Center for Microscopy and Imaging Research, a technology development center dedicated to technologies for bridging the resolution gap between light and electron microscopy, who passed away in Sept 2015. In this presentation, I will present an overview of Dr. Sosinsky's work using a variety of sophisticated multi-resolution imaging approaches developed at NCMIR to probe the trafficking, assembly and structure of gap junctions and a family of related proteins, the pannexins. Such techniques include genetically encoded tags for correlated light and electron microscopy, as well as widescale fluorescent brain mapping to plot the distribution of protein expression at high resolution across the brain. But Dr. Sosinsky also recognized that sophisticated imaging was not enough to create truly multidimensional views of cells and tissues. Rather, with the growing power of on-line data sharing and informatics platforms, researchers also had an obligation to collect and report their data in a manner that ensured it was viewable and usable by others. She was therefore a passionate advocate of data sharing, and together we worked on platforms and strategies for sharing and integrating multi-resolution imaging data. Through initiatives like the Neuroscience Information Framework (http://neuinfo.org) and the NIDDK Information Network, we saw the potential power of global search across publicly accessible data for creating models of cellular functions. Dr. Sosinsky ensured that all her data was part of these information networks, by making all her data available through public databases like Cell Centered Database (now part of the Cell Image Library Fig 1; http://www.cellimagelibrary.org). She also participated in efforts such as the Resource Identification Initiative (RII; Bandrowski et al., 2015), a grass roots effort to improve reporting standards for reagents and tools used to produce the findings of a study. The RII was launched because it was found that authors were not supplying enough information in their papers to conclusively identify which reagent or tool they used. In addition, even when the information was present, it required special text mining routines to extract. Because of these practices, it was impossible to ask a very basic question such as "what other studies were published using this reagent or tool?" The RII has worked with journals and authors to supply unique identifiers (accession numbers) for antibodies, genetically modified tools, databases and software tools in the material and methods sections of their papers. Since its launch in 2014, the RII has introduced these standards into > 90 journals, with over 650 articles appearing. Dr. Sosinsky's paper on pannexin distribution, which highlighted the variable results and difficulties in working with antibodies and genetically modified organisms, was actually the first paper to appear using RRID's. Her body of work not only represents elegant multi-scale imaging but serves as a model of how all science should be reported in the 21 st century. [3]

\section{References:}

[1] Bandrowski et al, F1000 Research, (2015) http://f1000research.com/articles/4-134/v2.

[2] Cone et al, Frontiers in Pharmacology, 4:6 (2013), http://dx.doi.org/10.3389/fphar.2013.00006 
[3] The author acknowledges funding from the National Institute on Drug Abuse for the Neuroscience Information Framework 1U24DA039832 and the National Institute of Diabetes, Digestive and Kidney Disease for the NIDDK Information Network 1U24DK097771

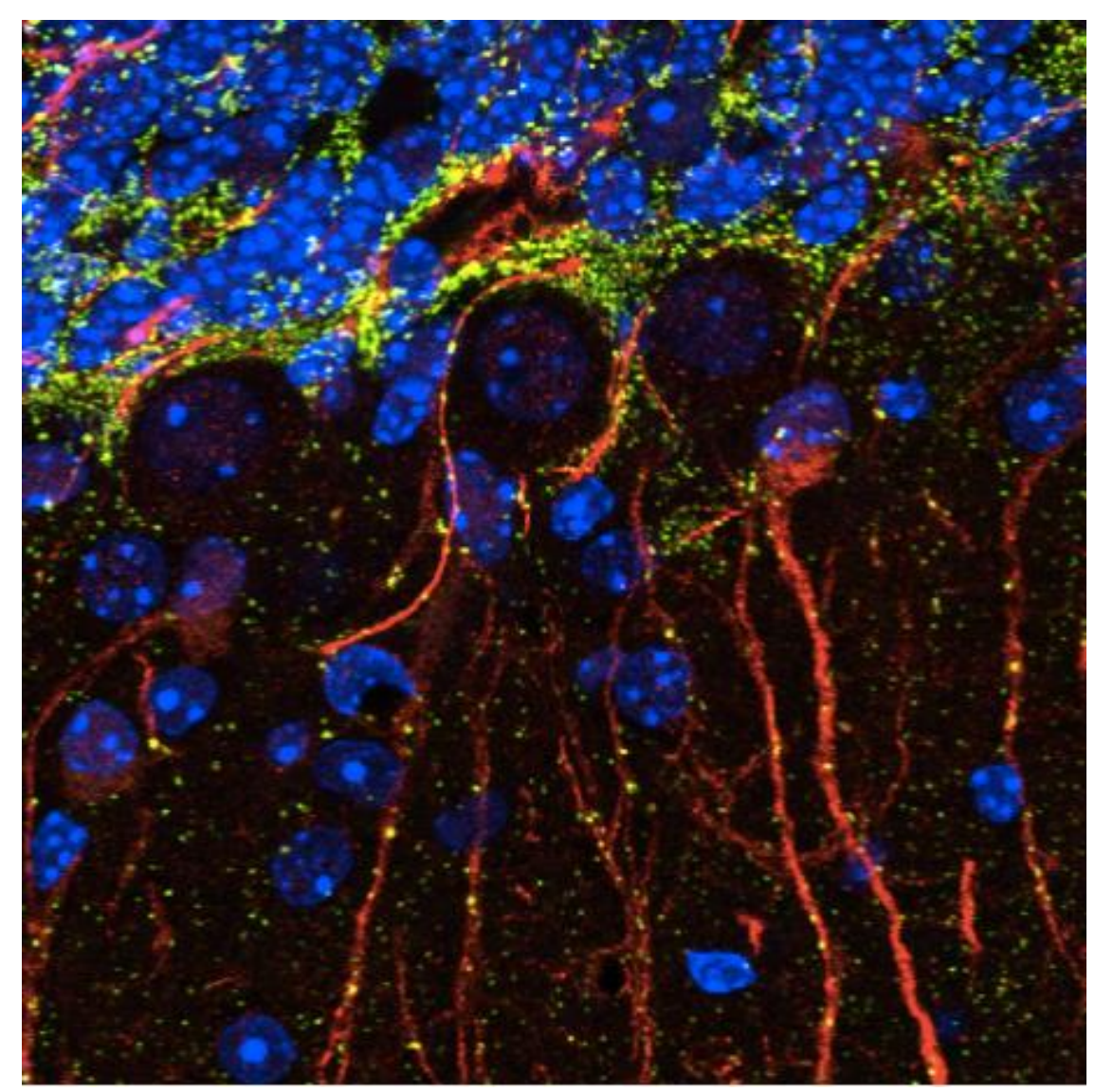

Figure 1. Single tile from a large scale brain mosaic, showing a projection of $4 \mathrm{Z}$-slices from 3 fluorescent channels acquired from brain tissue using a 2-photon confocal microscope. Red is Rhodamine RedX labeling of Chicken anti-GFAP. Green is Alexafluor488 labeling of Connexin43. Blue is DAPI staining of all nuclei, shared through CIL: http://www.cellimagelibrary.org/images/40197 